\title{
Hypoglycemia with New-Generation Basal Analog Insulins: A Descriptive Critical Review
}

\author{
Mazen Alsahli" ${ }^{*}$, James R Thrasher ${ }^{2}$ and John E Gerich ${ }^{3}$
}

${ }^{1}$ University of Toronto Faculty of Medicine, Toronto, Canada

${ }^{2}$ Arkansas Diabetes and Endocrinology Center, Little Rock, Arkansas, USA

${ }^{3}$ University of Rochester School of Medicine, Rochester, New York, USA

\begin{abstract}
Optimizing the treatment of people with diabetes relies on balancing the benefits of glycemic control with the risk of hypoglycemia. Although insulin is essential for treating patients with type 1 diabetes mellitus, patient and physician concerns regarding an increased risk of hypoglycemia can lead to delays in initiating insulin treatment in patients with type 2 diabetes mellitus. This clinical inertia contributes to reduced glycemic control and poorer outcomes for patients. Advances in insulin agents have reduced the risk of hypoglycemia. In particular, the introduction of insulin glargine, the first basal analog insulin with a 24-hour glucose-lowering profile with no pronounced peak, represented a significant step towards achieving this goal.

To further improve patient management, a number of insulin formulations and molecules are in development and are designed to have pharmacokinetic/pharmacodynamic (PK/PD) profiles allowing closer mimicking of normal physiologic insulin release. Here we review these new agents, and discuss their hypoglycemic risk as reported in clinical trials. In addition, the difficulties in making comparative evaluations from studies with different patient populations and definitions of hypoglycemia are discussed. Solutions to improve future clinical trials are suggested. In general, the improved PK/PD profiles of new-generation insulins appear to result in better clinical outcomes in terms of hypoglycemia. What is needed are head-to-head trials using standardized methods and criteria to allow clinicians to compare hypoglycemia rates between insulins, and help them to discuss appropriate choices of therapy with their patients.
\end{abstract}

Keywords: Hypoglycemia; Insulin; Type 1 diabetes; Type 2 diabetes

\section{Introduction}

Insulin treatment is essential for individuals with type 1 diabetes mellitus (T1DM). As a result of progressive beta-cell deterioration in type 2 diabetes mellitus (T2DM), most patients with T2DM eventually require insulin to achieve and maintain optimal glycemic targets. Usually, basal insulin is initiated before adding prandial therapy to maintain glycemic control [1]. Optimizing diabetes treatment depends on balancing glycemic control and the risk of hypoglycemia.

As plasma glucose levels decrease, there is a hierarchy of physiologic counterregulatory responses aimed at preventing further decreases and restoring normal plasma glucose levels (Figure 1) [2,3]. When plasma glucose levels decrease to $<70 \mathrm{mg} / \mathrm{dl}(3.8 \mathrm{mmol} / \mathrm{l})$, activation of counterregulation mechanisms begins; i.e., an increase in the secretion of glucagon, catecholamines, cortisol, and growth hormone, and a decrease in insulin secretion. These changes occur before there are any signs or symptoms related to hypoglycemia. As a consequence of these counterregulatory changes, there is an initial increase in hepatic and renal glucose release into the circulation (approximately equal amounts of glucose are released from the liver and kidneys), followed by a decrease in removal of glucose from the circulation. Decreases in plasma glucose to $\sim 60 \mathrm{mg} / \mathrm{dl}$ ( $3.3 \mathrm{mmol} / \mathrm{l})$ usually evoke the so-called autonomic warning symptoms (hunger, anxiety, palpitations, sweating, nausea). If interpreted correctly, these lead the patient to eat and thus prevent more serious hypoglycemia. If plasma glucose levels decrease to $\sim 55 \mathrm{mg} / \mathrm{dl}(3.0 \mathrm{mmol} / \mathrm{l})$, neuroglycopenic signs and symptoms of brain dysfunction (blurred vision, slurred speech, glassy eyed appearance, confusion) occur. Concentrations of plasma glucose below $30 \mathrm{mg} / \mathrm{dl}$ $(1.6 \mathrm{mmol} / \mathrm{l})$ - if prolonged - can cause seizures, permanent neurologic deficits, and death [4].

Within a few years of diabetes onset, people with T1DM develop impaired counterregulatory hormone responses, which are manifested first by decreased or absent glucagon responses to hypoglycemia [3]. This is followed by decreased catecholamine responses, and later by variable decreases in growth hormone and cortisol responses. Defective glucose counterregulation plays a major role in the susceptibility to severe hypoglycemia of people with T1DM. In contrast, people with T2DM experience more modest impairment in glucose counterregulation [5].

In addition to impaired glucose counterregulation, people with T1DM and T2DM may suffer from hypoglycemia unawareness [4]. These patients have an often transient loss of the autonomic symptoms warning them of developing hypoglycemia; these symptoms would normally have prompted them to take appropriate action (i.e., food intake before occurrence of severe hypoglycemia with neuroglycopenia). Hypoglycemia unawareness can be reversed in most cases by instigating a management plan that includes strict avoidance of hypoglycemia [6,7].

Although long-term studies suggest that tight glycemic control can reduce diabetes complications [8,9], this tight control increases the risk of hypoglycemia [9-11]. Concerns regarding hypoglycemia can lead to clinical inertia among physicians and barriers to initiating insulin

*Corresponding author: Mazen Alsahli, 531 Davis Dr, Newmarket, ON L3Y 6P5, Canada, Tel: +1 905-898-6100; E-mail: mazen.alsahli@utoronto.ca

Received June 05, 2015; Accepted June 29, 2015; Published July 03, 2015

Citation: Alsahli M, Thrasher JR, Gerich JE (2015) Hypoglycemia with NewGeneration Basal Analog Insulins: A Descriptive Critical Review. J Diabetes Metab 6: 576. doi:10.4172/2155-6156.1000576

Copyright: ( 2015 Alsahli M, et al. This is an open-access article distributed under the terms of the Creative Commons Attribution License, which permits unrestricted use, distribution, and reproduction in any medium, provided the original author and source are credited. 


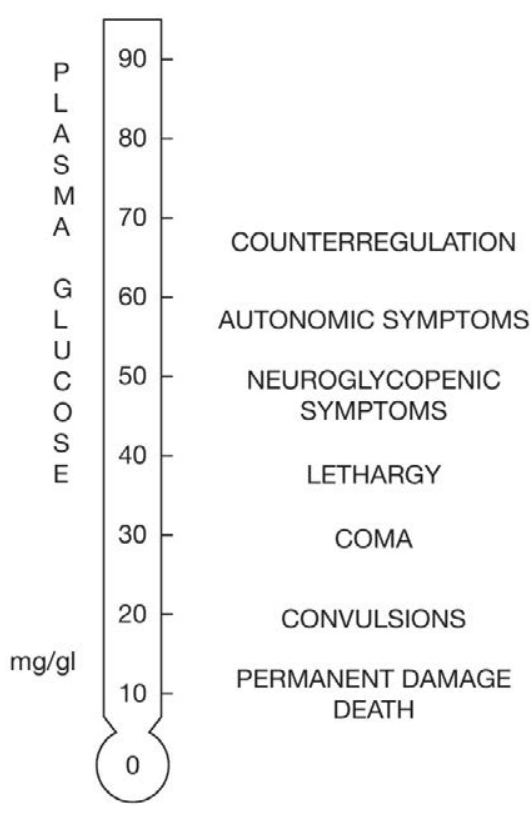

Figure 1: Consequences of hypoglycemia [3].

among patients. These have clinical consequences, as hypoglycemic events and the fear of future hypoglycemia are associated with reduced adherence to and persistence with treatment [12-15]. In turn, lower adherence is associated with the reduced likelihood to intensify treatment [16], and contributes to suboptimal glycemic control [17].

The majority of people with T2DM will progress to basal insulin therapy when oral antidiabetes drugs (OADs) fail to maintain adequate glycemic control. The relative simplicity of basal insulin regimens alongside the concept of "fix fasting first" makes basal insulin a desirable choice when intensifying treatment. However, as the disease progresses, this is usually insufficient for maintaining glycemic control and postprandial control is generally also required [18]. Various insulin therapies are available, but the introduction of the long-acting basal analog insulin glargine 100 units/ml (Gla-100) resulted in a reduction in rates of hypoglycemia compared with NPH insulin $[19,20]$. Diabetes treatment is an evolving field of medicine, with new-generation therapies in development.

The efficacy and safety of investigational insulins have been compared with standardized insulin in treat-to-target trials [21]. In these trials, insulin dosages are titrated in patients according to a specific algorithm so they can achieve a determined treatment glycemic goal [22]. At the same time, clinicians are able to determine differences in other treatment effects, like weight gain and hypoglycemia. It should be noted that the results of treat-to-target trials are sensitive to sample size [23]. For this reason, treat-to-target trials are subject to bias, unless a specific algorithm is rigorously enforced.

In this paper, we review the new generation of basal analog insulins in terms of their effect on hypoglycemia rates in clinical trials. A review of current literature and recent conference abstracts was undertaken to gather evidence. PubMed was searched with the search term: "basal insulin" OR "long-acting insulin" OR "ultra-long insulin" OR "longacting basal" OR "ultra-long acting basal". Furthermore, abstracts from the annual conferences of the European Association for the Study of Diabetes (EASD) of 2013 and 2014 were searched. Results were taken for any novel long-acting basal insulins with hypoglycemia data.

\section{Pharmacokinetic/Pharmacodynamic Profiles of Novel Basal Analog Insulins}

A key goal of insulin therapy is replicating physiologic basal insulin release: the release of insulin averages around $1.3 \mathrm{U} / \mathrm{h}$ under normoglycemic physiologic conditions [24]. As the first basal analog insulin with a 24-hour glucose-lowering profile with no pronounced peak [25], Gla-100 represented a significant step towards achieving this goal. Gla-100's pharmacokinetic (PK) and pharmacodynamic (PD) profile resulted in the first opportunity for basal insulin coverage with once-daily (QD) dosing. Insulin detemir (IDet) has a similar glucoselowering profile to Gla-100, often allowing basal coverage from a single daily dose, although twice-daily injections are required in up to $57 \%$ of patients (Figure 2) [26].

Advances in insulin therapy have included developing new agents or evolving established therapies. Increasing half-lives and improving peak-trough dynamics have provided a number of agents with therapeutic potential in treating hyperglycemia that more closely mimic physiologic insulin release patterns with fewer injections.

Insulin degludec (IDeg) is a new basal analog insulin with a PK/ PD profile that extends glucose lowering beyond 24 hours. Upon injection, IDeg di-hexamers assemble to form stable multi-hexamers. These form a soluble depot in the subcutaneous tissue from which IDeg monomers slowly dissociate. Studies to determine the PK/PD profile of IDeg demonstrate an evenly distributed glucose-lowering profile, with a terminal half-life of more than 25 hours under steady state conditions [27] and a duration of action reported to be over 40 hours [28].

A new formulation of Gla-100 is in development (insulin glargine 300 units/ml [Gla-300]), in which the same number of insulin units as Gla-100 is delivered, but in a third of the injection volume. Gla-100 forms crystals at neutral $\mathrm{pH}$ when injected subcutaneously; Gla-300 forms a more compact depot of crystals resulting in a lower depot surface area and a slower rate of dissolution. In patients with T1DM, PK/PD studies of Gla-300 have demonstrated a longer, smoother glucose-lowering profile compared with Gla-100, with a terminal halflife of approximately 19 hours and activity up to 36 hours, resulting in tighter glucose control [29-31]. The peak-trough ratio of Gla-300 is low at $\sim 1.7$ versus 2.3 for Gla-100, and this helps to minimize glycemic variability [32]. Theoretically, the less pronounced peak of action could result in a more gradual drop in blood glucose, with a reduced risk of hypoglycemia; this would need to be confirmed clinically in phase 3 trials.

Other basal insulins are in development that also have extended terminal half-lives and activity profiles. A PEGylated form of the fastacting basal insulin lispro LY2605541 (basal insulin peglispro [BIL]) has a functional size of approximately $75 \mathrm{kDa}$ due to the hydrodynamic properties of the polyethylene glycol chain linked to the insulin lispro

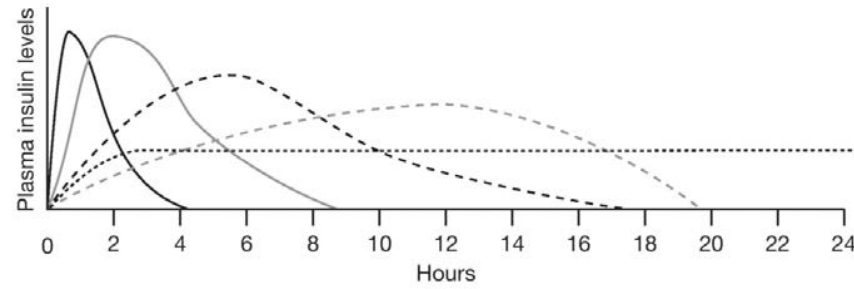

- Aspart, lispro, glulisine —-Regular - - NPH - - -Detemir ...... Glargine

Figure 2: Schematic showing pharmacokinetics of insulin therapies. 
Citation: Alsahli M, Thrasher JR, Gerich JE (2015) Hypoglycemia with New-Generation Basal Analog Insulins: A Descriptive Critical Review. J Diabetes Metab 6: 576. doi:10.4172/2155-6156.1000576

molecule. This results in slowed absorption and possible preferential hepatic activity [33]. BIL is approximately four times larger than unPEGylated lispro, which contributes to the PK/PD profile of BIL. Data from patients with T2DM suggest that a relatively long time is required to achieve steady state: 7-10 days versus 2-4 days for IDeg and Gla300 , respectively $[27,29]$. BIL has a terminal half-life of $45-76$ hours and low peak-trough fluctuation [34]. In a recent PK/PD study in patients with T2DM, the new insulin glargine LY2963016 was shown to have a similar profile to Gla-100, with a duration of action of approximately 24 hours [35].

In earlier-stage clinical development, HM12470 is a long-acting basal analog insulin produced by conjugating an insulin analog to the constant region of a human immunoglobulin fragment using a nonpeptidyl linker [36]. Animal studies in rats showed an approximately 43-hour half-life for HM12470 compared with 2.9 hours for IDeg [36]. Animal studies in mice, rats, and dogs showed a predicted half-life of HM12470 of approximately 132 hours and a low peak-trough ratio of 1.6 in humans [36]. This long half-life may allow once-weekly dosing.

BIOD-531 is a concentrated formulation of recombinant human insulin (400 units/ml) with a high dose:volume ratio and more rapid absorption, owing to the addition of EDTA, citrate, and $\mathrm{MgSO}_{4}$ [37]. Data suggest that BIOD-531 has a rapid onset of action and a duration of action of around 18 hours in non-diabetic obese subjects [38].

\section{New basal analog insulins and hypoglycemia}

As noted above, the clinical use of Gla-100 is associated with a lower risk of hypoglycemia than NPH insulin; this is a result of its longer, more constant $\mathrm{PK} / \mathrm{PD}$ profile and a reduction in the variability of glucose-lowering effects $[19,20]$. Data from clinical trials suggest that the newer generation of basal analog insulins, with their extended, smoother PK/PD profiles, may also result in improved rates of hypoglycemia compared with currently available insulins. In this section, we summarize data from clinical trials with these agents in patients with T2DM and T1DM (trials are summarized in Tables 1 and 2 , respectively). Data reviewed are restricted to head-to-head phase 3 clinical trials. Note that data on hypoglycemia rates for BIOD-531 and HM12470 are not yet available.

\section{Hypoglycemia in T2DM}

Hypoglycemia is a frequent adverse effect of the treatment of T2DM, with hypoglycemic events commonly occurring at night. As

\begin{tabular}{|c|c|c|c|c|c|c|c|}
\hline Study & $\begin{array}{l}\text { Investigational vs } \\
\text { comparator }\end{array}$ & Population & $\mathbf{n}$ & Hypoglycemia category & Result & $\mathrm{RR}^{\mathrm{a} / \mathrm{b}}(95 \% \mathrm{Cl})$ & $P$ value \\
\hline \multirow{3}{*}{$\begin{array}{l}\text { BEGIN Once } \\
\text { Long [46] }\end{array}$} & \multirow{3}{*}{$\begin{array}{l}\text { Insulin degludec vs } \\
\text { insulin glargine }\end{array}$} & \multirow{3}{*}{$\begin{array}{l}\text { Adults with T2DM (A1C 7.0- } \\
10.0 \% \text { ) taking OADs only }\end{array}$} & \multirow{3}{*}{1030} & $\begin{array}{l}\text { - Confirmed hypoglycemia } \\
\text { (PG } 54 \mathrm{mg} / \mathrm{dl} \text { or severe*) }\end{array}$ & $\begin{array}{l}1.52 \text { vs } 1.85 \\
\text { episodes/PYE }\end{array}$ & $\mathrm{RR}^{\mathrm{a}} 0.82(0.64-1.04)$ & 0.106 \\
\hline & & & & $\begin{array}{l}\text { - Nocturnal confirmed } \\
\text { hypoglycemia }\end{array}$ & $\begin{array}{l}0.25 \text { vs } 0.39 \\
\text { episodes/PYE }\end{array}$ & $\mathrm{RR}^{\mathrm{a}} 0.64(0.42-0.98)$ & 0.038 \\
\hline & & & & - Severe* & $\begin{array}{l}0.003 \text { vs } 0.023 \\
\text { episodes/PYE }\end{array}$ & $\operatorname{RR}^{a} 0.14(0.03-0.70)$ & 0.017 \\
\hline \multirow{3}{*}{$\begin{array}{l}\text { BEGIN Once Asia } \\
\text { [49] }\end{array}$} & \multirow{3}{*}{$\begin{array}{l}\text { Insulin degludec vs } \\
\text { insulin glargine }\end{array}$} & \multirow{3}{*}{$\begin{array}{l}\text { Asian adults with T2DM (A1C } \\
7.0-10.0 \% \text { ) taking OADs only }\end{array}$} & \multirow{3}{*}{435} & $\begin{array}{l}\text { - Confirmed hypoglycemia } \\
\text { (PG <54 mg/dl or severe*) }\end{array}$ & $\begin{array}{l}3.0 \text { vs } 3.7 \text { episodes/ } \\
\text { PYE }\end{array}$ & $\operatorname{RR}^{\mathrm{a}} 0.82(0.60-1.11)$ & 0.2 \\
\hline & & & & $\begin{array}{l}\text { - Nocturnal confirmed } \\
\text { hypoglycemia }\end{array}$ & $\begin{array}{l}0.8 \text { vs } 1.2 \text { episodes/ } \\
\text { PYE }\end{array}$ & $\mathrm{RR}^{\mathrm{a}} 0.62(0.38-1.04)$ & 0.07 \\
\hline & & & & - Severe* & - & - & - \\
\hline \multirow{3}{*}{$\begin{array}{l}\text { BEGIN Basal- } \\
\text { Bolus [47] }\end{array}$} & \multirow{3}{*}{$\begin{array}{l}\text { Insulin degludec } \\
+ \text { insulin aspart vs } \\
\text { insulin degludec + } \\
\text { insulin aspart }\end{array}$} & \multirow{3}{*}{$\begin{array}{l}\text { Adults with T2DM (A1C } \\
7.0-10.0 \% \text { ) on any insulin } \\
\text { regimen with or without } \\
\text { OADs }\end{array}$} & \multirow{3}{*}{1006} & $\begin{array}{l}\text { - Confirmed hypoglycemia } \\
\left(\mathrm{PG}<54 \mathrm{mg} / \mathrm{dl} \text { or severe }{ }^{\star}\right.\end{array}$ & $\begin{array}{l}11.1 \text { vs } 13.6 \\
\text { episodes/PYE }\end{array}$ & $\operatorname{RR}^{\mathrm{a}} 0.82(0.69-0.99)$ & 0.0359 \\
\hline & & & & $\begin{array}{l}\text { - Nocturnal confirmed } \\
\text { hypoglycemia }\end{array}$ & $\begin{array}{l}1.39 \text { vs } 1.84 \\
\text { episodes/PYE }\end{array}$ & $\operatorname{RR}^{\mathrm{a}} 0.75$ (0.58-0.99) & 0.0399 \\
\hline & & & & - Severe* & $\begin{array}{l}0.06 \text { vs } 0.05 \\
\text { episodes/PYE }\end{array}$ & - & - \\
\hline \multirow{5}{*}{ BEGIN Flex [48] } & \multirow{5}{*}{$\begin{array}{l}\text { Insulin degludec } \\
\text { flexible }^{\dagger} \text { vs insulin } \\
\text { degludec fixed vs } \\
\text { insulin glargine }\end{array}$} & \multirow{5}{*}{$\begin{array}{l}\text { Adults with T2DM taking } \\
\text { OADs (A1C } 7.0-11.0 \% \text { ) or } \\
\text { basal insulin + OADs (A1C } \\
7.0-10.0 \%)\end{array}$} & \multirow{5}{*}{687} & \multirow{2}{*}{$\begin{array}{l}\text { - Confirmed hypoglycemia } \\
\text { (PG } 54 \mathrm{mg} / \mathrm{dl} \text { or severe* }{ }^{*}\end{array}$} & \multirow{2}{*}{$\begin{array}{l}3.6 \text { vs } 3.6 \text { vs } 3.5 \\
\text { episodes/PYE }\end{array}$} & $\begin{array}{l}\mathrm{RR}^{\mathrm{a}} 1.03(0.75-1.40) \\
\text { flexible vs glargine }\end{array}$ & NS \\
\hline & & & & & & $\begin{array}{l}R^{a} 1.10(0.79-1.52) \\
\text { flexible vs fixed }\end{array}$ & NS \\
\hline & & & & \multirow{2}{*}{$\begin{array}{l}\text { - Nocturnal confirmed } \\
\text { hypoglycemia }\end{array}$} & \multirow{2}{*}{$\begin{array}{l}0.6 \text { vs } 0.6 \text { vs } 0.8 \\
\text { episodes/PYE }\end{array}$} & $\begin{array}{l}R^{\mathrm{a}} 0.77(0.44-1.35) \\
\text { flexible vs glargine }\end{array}$ & NS \\
\hline & & & & & & $\begin{array}{l}R^{\mathrm{R}} 1.18(0.66-2.12) \\
\text { flexible vs fixed }\end{array}$ & NS \\
\hline & & & & - Severe* & - & - & - \\
\hline \multirow{3}{*}{ EDITION 1 [54] } & \multirow{3}{*}{ Gla-300 vs Gla- 100} & \multirow{3}{*}{$\begin{array}{l}\text { Adults with T2DM (A1C } \\
7.0-10.0 \% \text { ) using } \geq 42 \mathrm{U} / \\
\text { day basal insulin + mealtime } \\
\text { insulin }\end{array}$} & \multirow{3}{*}{807} & $\begin{array}{l}\text { - Confirmed hypoglycemia } \\
\left.\text { (PG } \leq 70 \mathrm{mg} / \mathrm{dl} \text { or severe }{ }^{\star}\right)\end{array}$ & $81.9 \%$ vs $87.8 \%$ & $\mathrm{RR}^{\mathrm{b}} 0.93(0.88-0.99)$ & - \\
\hline & & & & $\begin{array}{l}\text { - Nocturnal confirmed } \\
\text { hypoglycemia }\end{array}$ & $44.6 \%$ vs $57.5 \%$ & $\mathrm{RR}^{\mathrm{b}} 0.78(0.68-0.89)$ & - \\
\hline & & & & - Severe* & $5.0 \%$ vs $5.7 \%$ & $\mathrm{RR}^{\mathrm{b}} 0.87(0.48-1.55)$ & - \\
\hline \multirow[t]{3}{*}{ EDITION 2 [55] } & \multirow[t]{3}{*}{ Gla-300 vs Gla- 100} & \multirow{3}{*}{$\begin{array}{l}\text { Adults with T2DM (A1C } \\
7.0-10.0 \% \text { ) using } \geq 42 \mathrm{U} / \text { day } \\
\text { basal insulin + OADs }\end{array}$} & \multirow[t]{3}{*}{811} & $\begin{array}{l}\text { - Confirmed hypoglycemia } \\
\text { (PG } \leq 70 \mathrm{mg} / \mathrm{dl} \text { or severe* }\end{array}$ & $70.0 \%$ vs $77.3 \%$ & $\mathrm{RR}^{\mathrm{b}} 0.90(0.83-0.98)$ & - \\
\hline & & & & $\begin{array}{l}\text { - Nocturnal confirmed } \\
\text { hypoglycemia }\end{array}$ & $28.3 \%$ vs $39.9 \%$ & $\operatorname{RR}^{\mathrm{b}} 0.71(0.58-0.86)$ & - \\
\hline & & & & - Severe* & $1.0 \%$ vs $1.5 \%$ & - & - \\
\hline
\end{tabular}


Citation: Alsahli M, Thrasher JR, Gerich JE (2015) Hypoglycemia with New-Generation Basal Analog Insulins: A Descriptive Critical Review. J Diabetes Metab 6: 576. doi:10.4172/2155-6156.1000576

Page 4 of 9

\begin{tabular}{|c|c|c|c|c|c|c|c|}
\hline \multirow{3}{*}{ EDITION 3 [56] } & \multirow{3}{*}{ Gla-300 vs Gla- 100} & \multirow{3}{*}{$\begin{array}{l}\text { Adults with T2DM (A1C 7.0- } \\
11.0 \% \text { ) on OADs }\end{array}$} & \multirow{3}{*}{878} & $\begin{array}{l}\text { - Confirmed hypoglycemia } \\
\text { (PG } \leq 70 \mathrm{mg} / \mathrm{dl} \text { or severe } \text { sever }^{\star}\end{array}$ & $46.2 \%$ vs $52.5 \%$ & $\mathrm{RR}^{\mathrm{b}} 0.88(0.77-1.01)$ & - \\
\hline & & & & $\begin{array}{l}\text { - Nocturnal confirmed } \\
\text { hypoglycemia }\end{array}$ & $17.9 \%$ vs $23.5 \%$ & $R^{b} 0.76(0.59-0.99)$ & - \\
\hline & & & & - Severe* & $0.9 \%$ vs $0.9 \%$ & - & - \\
\hline \multirow{3}{*}{ EDITION JP 2 [57] } & \multirow{3}{*}{ Gla-300 vs Gla- 100} & \multirow{3}{*}{$\begin{array}{l}\text { Asian adults with T2DM } \\
\text { basal insulin + OADs }\end{array}$} & \multirow{3}{*}{241} & $\begin{array}{l}\text { - Confirmed hypoglycemia } \\
\text { (PG } \leq 70 \mathrm{mg} / \mathrm{dl} \text { or severe* }\end{array}$ & $65.0 \%$ vs $76.7 \%$ & $\mathrm{RR}^{\mathrm{b}} 0.86(0.73-1.01)$ & - \\
\hline & & & & $\begin{array}{l}\text { - Nocturnal confirmed } \\
\text { hypoglycemia }\end{array}$ & $28.3 \%$ vs $45.8 \%$ & $\mathrm{RR}^{\mathrm{b}} 0.62(0.44-0.88)$ & - \\
\hline & & & & - Severe* & - & - & - \\
\hline \multirow{2}{*}{$\begin{array}{l}\text { Bergenstal et al., } \\
2012 \text { [58] }\end{array}$} & \multirow{2}{*}{$\begin{array}{l}\text { peglispro vs insulin } \\
\text { glargine }\end{array}$} & \multirow{2}{*}{$\begin{array}{l}\text { Adults with T2DM (A1C } \\
\leq 10.5 \% \text { ) on basal insulin and } \\
\text { OADs }\end{array}$} & \multirow{2}{*}{289} & $\begin{array}{l}\text { - Total hypoglycemia (BG } \leq 70 \\
\mathrm{mg} / \mathrm{dl} \text { or symptoms) }\end{array}$ & $\begin{array}{l}1.34 \text { vs } 0.34 \\
\text { events/30 days }\end{array}$ & - & 0.804 \\
\hline & & & & - Nocturnal hypoglycemia & $\begin{array}{l}0.25 \text { vs } 0.39 \\
\text { events } / 30 \text { days }\end{array}$ & - & 0.178 \\
\hline ELEMENT 2 [61] & $\begin{array}{l}\text { LY2963016 vs } \\
\text { insulin glargine }\end{array}$ & $\begin{array}{l}\text { Adults with T2DM on OADS } \\
\text { (A1C } 7.0-11.0 \% \text { ) or insulin } \\
\text { glargine + OADs (A1C } \\
\leq 11.0 \% \text { ) }\end{array}$ & 756 & $\begin{array}{l}\text { - Total hypoglycemia (BG } \leq 70 \\
\mathrm{mg} / \mathrm{dl} \text { or symptoms) }\end{array}$ & 21.3 vs 22.3 & - & 0.995 \\
\hline
\end{tabular}

*Requiring assistance; ${ }^{\dagger}$ dosing schedule creating $8-40$ hours between injections.

A1C, glycated hemoglobin; BG, blood glucose; Cl, confidence interval; NS, not significant; PG, plasma glucose; PYE, patient year of exposure; OADs, oral antidiabetic drugs; $R^{a}$, rate ratio; $R^{b}$, relative risk; T2DM, type 2 diabetes mellitus.

Table 1: Hypoglycemia in randomized clinical trials of novel basal analog insulins in T2DM [46-49,54-58,61].

\begin{tabular}{|c|c|c|c|c|c|c|c|}
\hline Study & $\begin{array}{l}\text { Investigational vs } \\
\text { comparator }\end{array}$ & Population & n & Hypoglycemia category & Result & $\mathrm{RR}^{\mathrm{a} / \mathrm{b}}(95 \% \mathrm{Cl})$ & $P$ value \\
\hline \multirow{3}{*}{$\begin{array}{l}\text { BEGIN Basal- } \\
\text { Bolus Type } 1 \text { [28] }\end{array}$} & \multirow{3}{*}{$\begin{array}{l}\text { Insulin degludec + insulin } \\
\text { aspart vs insulin degludec } \\
+ \text { insulin aspart }\end{array}$} & \multirow{3}{*}{$\begin{array}{l}\text { Adults with T1DM (A1C } \\
\leq 10 \% \text { ) on basal-bolus } \\
\text { therapy }\end{array}$} & \multirow{3}{*}{629} & $\begin{array}{l}\text { - Confirmed hypoglycemia (PG } \\
<54 \mathrm{mg} / \mathrm{dl} \text { or severe }{ }^{\star} \text { ) }\end{array}$ & $\begin{array}{l}42.54 \text { vs } 40.18 \\
\text { events/PYE }\end{array}$ & $\mathrm{RR}^{\mathrm{a}} 1.07$ (0.89-1.28) & 0.48 \\
\hline & & & & $\begin{array}{l}\text { - Nocturnal confirmed } \\
\text { hypoglycemia }\end{array}$ & $\begin{array}{l}4.41 \text { vs } 5.86 \\
\text { events/PYE }\end{array}$ & $\mathrm{RR}^{\mathrm{a}} 0.75(0.59-0.96)$ & 0.021 \\
\hline & & & & - Severe* & $\begin{array}{l}0.21 \text { vs } 0.16 \\
\text { events/PYE }\end{array}$ & $\operatorname{RR}^{\mathrm{a}} 1.38(0.72-2.64)$ & 0.34 \\
\hline \multirow{6}{*}{$\begin{array}{l}\text { BEGIN Flex T1 } \\
{[62]}\end{array}$} & \multirow{6}{*}{$\begin{array}{l}\text { Insulin degludec flexible } \\
+ \text { insulin aspart vs } \\
\text { insulin degludec fixed + } \\
\text { insulin aspart vs insulin } \\
\text { glargine+insulin aspart }\end{array}$} & \multirow{6}{*}{$\begin{array}{l}\text { Adults with T1DM (A1C } \\
\leq 10 \% \text { ) on basal-bolus } \\
\text { therapy }\end{array}$} & \multirow{6}{*}{493} & \multirow{2}{*}{$\begin{array}{l}\text { - Confirmed hypoglycemia (PG } \\
<54 \text { mg/dl or severe* }\end{array}$} & \multirow{2}{*}{$\begin{array}{l}82.4 \text { vs } 88.3 \text { vs } \\
79.7 \text { events/PYE }\end{array}$} & $\begin{array}{l}\mathrm{RR}^{\mathrm{a}} 1.03(0.85-1.26) \\
\text { flexible vs glargine }\end{array}$ & NS \\
\hline & & & & & & $\begin{array}{l}\mathrm{RR}^{\mathrm{a}} 0.92(0.76-1.12) \\
\text { flexible vs fixed }\end{array}$ & NS \\
\hline & & & & \multirow{2}{*}{$\begin{array}{l}\text { - Nocturnal confirmed } \\
\text { hypoglycemia }\end{array}$} & \multirow{2}{*}{$\begin{array}{l}6.2 \text { vs } 9.6 \text { vs } 10.0 \\
\text { events/PYE }\end{array}$} & $\begin{array}{l}\mathrm{RR}^{\mathrm{a}} 0.60(0.44-0.82) \\
\text { flexible vs glargine }\end{array}$ & 0.001 \\
\hline & & & & & & $\begin{array}{l}\mathrm{RR}^{\mathrm{a}} 0.63(0.46-0.86) \\
\text { flexible vs fixed }\end{array}$ & 0.003 \\
\hline & & & & \multirow{2}{*}{ - Severe* } & \multirow{2}{*}{$\begin{array}{l}0.3 \text { vs } 0.4 \text { vs } 0.5 \\
\text { events/PYE }\end{array}$} & $\begin{array}{l}\mathrm{RR}^{\mathrm{a}} 0.89(0.40-1.99) \\
\text { flexible vs glargine }\end{array}$ & NS \\
\hline & & & & & & $\begin{array}{l}\mathrm{RR}^{\mathrm{a}} 1.09(0.48-2.48) \\
\text { flexible vs fixed }\end{array}$ & NS \\
\hline \multirow[t]{3}{*}{ EDITION 4 [63] } & \multirow[t]{3}{*}{ Gla-300 vs Gla-100 } & \multirow{3}{*}{$\begin{array}{l}\text { Adults with T1DM basal } \\
\text { insulin + prandial insulin }\end{array}$} & \multirow{3}{*}{549} & $\begin{array}{l}\text { - Confirmed hypoglycemia (PG } \\
\leq 70 \mathrm{mg} / \mathrm{dl} \text { or severe*) }\end{array}$ & $\begin{array}{l}78.4 \text { vs } 72.5 \\
\text { events/PYE }\end{array}$ & $\operatorname{RR}^{\mathrm{a}} 1.09$ (0.94-1.25) & - \\
\hline & & & & $\begin{array}{l}\text { - Nocturnal confirmed } \\
\text { hypoglycemia }\end{array}$ & $\begin{array}{l}8.0 \text { vs } 8.9 \text { events/ } \\
\text { PYE }\end{array}$ & $\mathrm{RR}^{\mathrm{a}} 0.90(0.71-1.14)$ & - \\
\hline & & & & - Severe ${ }^{*}$ & $6.6 \%$ vs $9.5 \%$ & - & - \\
\hline \multirow[t]{3}{*}{$\begin{array}{l}\text { Edition } 4 \text { JP } 1 \\
\text { [64] }\end{array}$} & Gla300 vs Gla-100 & $\begin{array}{l}\text { Adults with T1DM basal } \\
\text { insulin + prandial insulin }\end{array}$ & 243 & $\begin{array}{l}\text { - Confirmed hypoglycemia (PG } \\
\leq 70 \mathrm{mg} / \mathrm{dl} \text { or severe* }\end{array}$ & $96.7 \%$ vs $97.5 \%$ & $\mathrm{RR}^{\mathrm{b}} 0.99(0.95-1.04)$ & - \\
\hline & & & & $\begin{array}{l}\text { - Nocturnal confirmed } \\
\text { hypoglycemia }\end{array}$ & $68.9 \%$ vs $81.0 \%$ & $\mathrm{RR}^{\mathrm{b}} 0.85(0.73-0.99)$ & - \\
\hline & & & & - Severe* & - & - & - \\
\hline
\end{tabular}




\begin{tabular}{|c|c|c|c|c|c|c|c|}
\hline \multirow{3}{*}{$\begin{array}{l}\text { Rosenstock et } \\
\text { al., } 2013 \text { [65] }\end{array}$} & \multirow{3}{*}{$\begin{array}{l}\text { LY2605541 + prandial } \\
\text { insulin vs insulin glargine } \\
+ \text { prandial insulin }\end{array}$} & \multirow{3}{*}{$\begin{array}{l}\text { Adults with T1DM (A1C } \\
\leq 10.5 \%) \text { on basal-bolus } \\
\text { insulin }\end{array}$} & \multirow{3}{*}{137} & $\begin{array}{l}\text { - Total hypoglycemia (BG } \leq 70 \\
\mathrm{mg} / \mathrm{dl} \text { or symptoms) }\end{array}$ & $\begin{array}{l}8.74 \text { vs } 7.36 \\
\text { events/PME }\end{array}$ & $\mathrm{RR}^{\mathrm{a}} 1.12(1.03-1.23)$ & 0.037 \\
\hline & & & & - Nocturnal hypoglycemia & $\begin{array}{l}0.88 \text { vs } 1.13 \\
\text { events/PME }\end{array}$ & $\mathrm{RR}^{\mathrm{a}} 0.75(0.62-0.90)$ & 0.012 \\
\hline & & & & - Severe & $\begin{array}{l}6 \text { events in both } \\
\text { arms (in } 5 \text { vs } 3 \\
\text { patients) }\end{array}$ & - & - \\
\hline ELEMENT 1 [66] & $\begin{array}{l}\text { LY2963016 + insulin } \\
\text { lispro vs insulin glargine + } \\
\text { insulin lispro }\end{array}$ & $\begin{array}{l}\text { Adults with T1DM (A1C } \\
\leq 11 \% \text { ) on basal-bolus } \\
\text { insulin }\end{array}$ & 535 & $\begin{array}{l}\text { - Total hypoglycemia (BG } \\
\leq 70 \mathrm{mg} / \text { dl or symptoms) }\end{array}$ & $86.5 \%$ vs $89.2 \%$ & - & 0.717 \\
\hline
\end{tabular}

significant; PG, plasma glucose; PME, patient month of exposure; PYE, patient year of exposure; RRa, rate ratio; RRb, relative risk; T1DM, type 1 diabetes mellitus.

Table 2: Hypoglycemia in randomized clinical trials of novel basal analog insulins in T1DM [28,62-66].

well as accidents and physical injury, the sequelae of hypoglycemia involve neurologic and cardiovascular complications discussed above [39]. Although reported rates of hypoglycemia in clinical trials vary considerably because of differences in study design, definitions used, and population included (among other factors) [40], a recent nonrandomized retrospective study using real-world outcomes from patients treated with Gla-100 or IDet in the United States suggested a rate of hypoglycemia of $\sim 8 \%$ [41]. Treatment regimen and a history of hypoglycemia are the most important predictors of future hypoglycemic events, but renal insufficiency, older age, and history of hypoglycemiaassociated autonomic failure are also important risk factors [42].

Insulin degludec: IDeg is currently licensed for use in the European Union, Japan, Mexico, and India. However, to date the US Food and Drug Administration (FDA) has declined to approve IDeg following a possible signal of an increased risk of major adverse cardiovascular events, and has requested additional cardiovascular outcomes data from a dedicated trial $[43,44]$.

In common with the other novel basal analog insulins, head-tohead trials of IDeg have concentrated on Gla-100 as a comparator, as this is the most widely used and extensively studied basal analog insulin. A meta-analysis of results from phase 3 trials suggests that the efficacy, in terms of lowering glycated hemoglobin (A1C), of IDeg and Gla-100 was similar in patients with T2DM [45]. Hypoglycemia event outcomes with IDeg compared with Gla-100 have been reported from the BEGIN series of phase 3 trials, in which confirmed hypoglycemia was defined as a composite of episodes with a recorded self-monitored blood glucose (SMBG) value of less than $\sim 54 \mathrm{mg} / \mathrm{dl}(3.1 \mathrm{mmol} / \mathrm{l})$ and severe episodes were defined as those requiring assistance. Statistical comparisons of hypoglycemia between patient groups primarily used rate ratios in the trials.

The BEGIN Once Long trial was a 52-week, parallel-group, openlabel, head-to-head trial of Gla-100 or IDeg QD with treat-to-target titration to achieve pre-breakfast plasma glucose of $\sim 70-90 \mathrm{mg} / \mathrm{dl}$ (3.9-4.9 mmol/l) [46]. In this trial, rates of confirmed hypoglycemia were similar between patient groups, and rates of confirmed nocturnal hypoglycemia (events occurring between 00:01 and 5:59 hours) were significantly lower with IDeg (Table 1). Although severe hypoglycemic episodes were rare in both groups, there was a statistical advantage for patients treated with IDeg (Table 1).

The BEGIN Basal-Bolus trial was a 52-week, parallel-group, openlabel trial of patients treated with IDeg or Gla-100 QD plus mealtime insulin aspart, in which basal analog insulins were titrated with an aim of achieving blood glucose $\sim 70-90 \mathrm{mg} / \mathrm{dl}$ (3.9-5.0 mmol/l) [47]. In this study, both confirmed hypoglycemia and confirmed nocturnal hypoglycemic events were significantly fewer with IDeg (Table 1). The incidence of severe episodes of hypoglycemia appeared to be similar between groups; however, rates were too low to assess statistically (Table 1).

The BEGIN series of studies also included trials with a 26 -week duration. BEGIN FLEX was a 26-week, open-label, three-arm, parallelgroup trial, in which patients received IDeg QD flexibly dosed to a prespecified rotating morning and evening dosing schedule (IDeg FLEX), creating 8-40 hour intervals between doses [48]. IDeg QD was dosed at the evening meal, or Gla-100 dosed at the same time each day. This trial had a treat-to-target design aimed at achieving blood glucose $\sim 70-90$ $\mathrm{mg} / \mathrm{dl}$ (3.9-5.0 $\mathrm{mmol} / \mathrm{l})$ [48]. There were no significant differences in terms of confirmed hypoglycemia, confirmed nocturnal hypoglycemia, and/or severe hypoglycemia between the three treatment groups (Table $1)$.

In the 26-week, open-label BEGIN Once Asia study, patients were treated with either IDeg or Gla-100 QD, with a titration target of blood glucose $\sim 70-90 \mathrm{mg} / \mathrm{dl}$ (3.9-5.0 mmol/l) [49]. There were no significant differences in rates of confirmed overall or nocturnal hypoglycemia over the trial period (Table 1).

Insulin glargine 300 units/ml: In February 2015, the FDA approved Gla-300 based on data from the EDITION series of 26week, phase 3 clinical trials [50]. Around the same time, the European Medicines Agency (EMA) adopted a positive opinion towards Gla-300 [51]. A meta-analysis of the currently available data from the EDITION trials of Gla-300 versus Gla-100 showed similar reductions in A1C (least square [LS] mean change -1.02\%) for both formulations [52].

The main secondary outcome in the EDITION 1, 2, and 3 trials was the percentage of participants with one or more confirmed nocturnal hypoglycemic events, defined as a composite of events with an SMBG value $\leq 70 \mathrm{mg} / \mathrm{dl}(3.9 \mathrm{mmol} / \mathrm{l})$ [53] or a severe event (requiring assistance) occurring between 00:00 and 05:59 hours from Week 9 to Week 26 of treatment. Confirmed or severe hypoglycemia events at any time of the night were also assessed over the full 26-week study period and for the first 8 weeks of the study.

EDITION 1 was a randomized, open-label, parallel-group trial in which patients using high daily doses of basal insulin ( $\geq 42 \mathrm{U} /$ day) alongside mealtime insulin received Gla-300 or Gla-100 QD titrated to achieve fasting plasma glucose (FPG) $\sim 80-100 \mathrm{mg} / \mathrm{dl}(4.4-5.6 \mathrm{mmol} / \mathrm{l})$ [54]. There was a significantly lower incidence of confirmed (plasma glucose $\leq 70 \mathrm{mg} / \mathrm{dl}$ ) or severe nocturnal hypoglycemic events in the Gla-300 group compared with the Gla-100 group between Week 9 and Week 26 ( $36 \%$ vs $46 \%$, respectively; $P=0.0045$ ) as well as over the full 26-week trial period (Table 1) [54]. Over the full 26-week trial period, the incidence of confirmed hypoglycemia (plasma glucose $\leq 70 \mathrm{mg} / \mathrm{dl}$ ) was also lower in the Gla-300 group, while there was no statistically significant difference in the incidence of severe hypoglycemic events (Table 1). 
In the EDITION 2 trial, a randomized, open-label, parallel-group study, adults treated with high-dose basal insulin ( $\geq 42 \mathrm{U} /$ day) and OADs received either Gla-300 or Gla-100 QD titrated to an FPG target of $\sim 80-100 \mathrm{mg} / \mathrm{dl}(4.4-5.6 \mathrm{mmol} / \mathrm{l})$ [55]. The incidence of confirmed (plasma glucose $\leq 70 \mathrm{mg} / \mathrm{dl}$ ) or severe nocturnal hypoglycemic events was significantly lower in the Gla-300 group than the Gla-100 group between Week 9 and Week 26 (21.6\% vs $27.9 \%$, respectively; $P=0.038$ ), as was the incidence over the full study period (Table 1) [55]. The incidence of confirmed (plasma glucose $\leq 70 \mathrm{mg} / \mathrm{dl}$ ) or severe hypoglycemic events occurring at any time was lower in the Gla-300 group than the Gla-100 group during the full 26-week study period, similarly to EDITION 1 (Table 1). The incidence of severe hypoglycemia was low in both groups over the 26-week period (Table 1).

In the open-label EDITION 3 trial, insulin-naïve participants on OADs were randomized to receive Gla-300 or Gla-100 titrated to an FPG target of $\sim 80-100 \mathrm{mg} / \mathrm{dl}$ (4.4-5.6 mmol/l). There was no significant difference in the incidence of confirmed (plasma glucose $<70 \mathrm{mg} / \mathrm{dl}$ ) or severe nocturnal hypoglycemia between Week 9 and Week $26(P=0.45)$ [56]. Over the full 26-week period, the incidence of confirmed (plasma glucose $<70 \mathrm{mg} / \mathrm{dl}$ ) or severe nocturnal hypoglycemia was lower in the Gla-300 group than the Gla-100 group, and the incidence of confirmed (plasma glucose $<70 \mathrm{mg} / \mathrm{dl}$ ) or severe hypoglycemia at any time was numerically lower in the Gla-300 group (Table 1). There was no difference in the incidence of severe hypoglycemia over the whole treatment period between groups (Table 1).

Gla-300 has also been compared with Gla-100 in the EDITION JP 2 trial, an open-label study in Japanese patients using basal insulin and OADs [57]. In this study, no main secondary endpoint was defined. However, although not powered to identify statistical differences in hypoglycemia, the incidence of confirmed (plasma glucose $<70 \mathrm{mg} / \mathrm{dl}$ ) or severe nocturnal hypoglycemia was lower in the Gla-300 group than in the Gla-100 group ( $25.4 \%$ vs $43.7 \%$; relative risk $0.58,95 \%$ CI 0.40 0.85 ) [57]. Confirmed (plasma glucose $<70 \mathrm{mg} / \mathrm{dl}$ ) or severe nocturnal hypoglycemia was also lower over the full 26-week study period; confirmed (plasma glucose $<70 \mathrm{mg} / \mathrm{dl}$ ) or severe hypoglycemia at any time was numerically lower in the Gla-300 group (Table 1), and severe hypoglycemia was low in both groups $(2.5 \%$ and $1.7 \%$ with Gla-300 and Gla-100, respectively).

A meta-analysis of the EDITION 1, 2, and 3 trials showed a reduction in overall confirmed or severe hypoglycemia when using Gla-300 compared with Gla-100, which was not consistently observed in the individual clinical trials included in the meta-analysis [52].

LY2605541, basal insulin peglispro: Data on hypoglycemia in patients receiving BIL are available only from a single phase 2 trial in T2DM [58]. In this 12-week, open-label, three-arm, parallelgroup study, patients were treated with basal insulin and OADs and randomized to receive either BIL or Gla-100 QD. Hypoglycemia was defined as any event with a blood glucose measurement $\leq 70 \mathrm{mg} / \mathrm{dl}$ ( $3.9 \mathrm{mmol} / \mathrm{l})$, and severe hypoglycemia was defined as that requiring assistance from another person with prompt recovery in response to carbohydrate intake. Insulin dose was titrated to a blood glucose target of $\leq 100 \mathrm{mg} / \mathrm{dl}(\leq 5.6 \mathrm{mmol} / \mathrm{l})$. The efficacy of BIL was similar to that of Gla-100 in terms of $\mathrm{A} 1 \mathrm{C}$ reduction and there were no significant differences in terms of incidence of total or nocturnal hypoglycemia events (Table 1). However, when results were adjusted for baseline hypoglycemia, a significant $48 \%$ reduction favoring BIL was detected for nocturnal hypoglycemia $(P=0.021)$ [58]. There were no severe hypoglycemic events reported during the study period.
Four phase 3 trials comparing BIL to Gla-100 or NPH have been completed as part of the IMAGINE trial series; reporting of results is expected shortly (NCT01582451, NCT01790438, NCT01435616, NCT01468987; https://clinicaltrials.gov)

New insulin glargine LY2963016: The EMA recommended approval of LY2963016 as a biosimilar in June 2014 [59], and the FDA tentatively approved the New Drug Application for LY2963016 in August 2014 [60].

In the ELEMENT 2 trial, a 26-week, phase 3, double-blind, parallelgroup study, insulin-naïve patients treated with OADs received either LY2963016 or Gla-100 QD. The insulin dose was titrated to achieve blood glucose $\leq 100 \mathrm{mg} / \mathrm{dl}(\leq 5.6 \mathrm{mmol} / \mathrm{l})$. The efficacy of both agents in terms of A1C reduction was similar [61]. With regard to rate of total hypoglycemia (defined as blood glucose $\leq 70 \mathrm{mg} / \mathrm{dl}$ [3.9 $\mathrm{mmol} / \mathrm{l}]$ ), where measures were available, there was no statistically significant difference between LY2963016 and Gla-100 (Table 1).

\section{Hypoglycemia in T1DM}

The incidence of hypoglycemia in patients with T1DM is generally higher than among those with T2DM. Adults with T1DM have $\sim 2$ episodes of mild hypoglycemia per week; the annual prevalence of severe hypoglycemia is $\sim 30 \%$, with several factors, such as long disease duration, increasing its incidence $[39,40]$.

Insulin degludec: In their meta-analysis of phase 3 trials of IDeg versus Gla-100, Vora et al. reported that the two agents had similar efficacy in terms of reduction of A1C in T1DM patients [45].

In the BEGIN Basal-Bolus Type 1 study, a 52-week, parallel-group, phase 3 study, T1DM patients previously treated with basal-bolus insulin for $\geq 1$ year received either IDeg or Gla-100 QD with mealtime insulin aspart [28]. Both basal and mealtime insulin were titrated to achieve blood glucose $\sim 70-90 \mathrm{mg} / \mathrm{dl}$ (3.9-5.0 mmol/l). In this study, rates of confirmed hypoglycemia were similar between patient groups, confirmed nocturnal hypoglycemia was significantly lower with IDeg than with Gla-100, and a similar rate of severe hypoglycemia was observed for both treatment groups (Table 2).

In the BEGIN FLEX T1 trial, patients received mealtime insulin aspart alongside basal analog insulin treatment. The trial had a treat-totarget design with basal analog insulin titrated to achieve blood glucose $\sim 70-90 \mathrm{mg} / \mathrm{dl}(4.0-5.0 \mathrm{mmol} / \mathrm{l})$ and mealtime insulin titrated to achieve $\leq 90 \mathrm{mg} / \mathrm{dl}$ ( $\leq 5.0 \mathrm{mmol} / \mathrm{l})$, based on the preceding day's pre-lunch, predinner, and bedtime SMPG values [62]. After 26 weeks, confirmed hypoglycemia rates were similar and rates of severe events were low in all groups (Table 2). Confirmed nocturnal events were significantly lower with the IDeg FLEX dosage compared with either IDeg (37\%, $P=0.003)$ or Gla-100 (40\%, $P=0.001)[62]$.

Insulin glargine $\mathbf{3 0 0}$ units $/ \mathbf{m l}$ : Two phase 3 studies have been conducted in otherwise healthy patients with T1DM as part of the EDITION series of clinical trials. Study designs, definitions of hypoglycemia, and titration targets were consistent throughout the series in patients with T2DM and T1DM.

The EDITION 4 trial was a 26-week, open-label study in which participants were randomized to Gla-300 (morning or evening) or Gla-100 (morning or evening) while continuing their mealtime insulin [63]. Gla-300 showed similar efficacy to Gla-100 in terms of lowering A1C. Confirmed (plasma glucose $<70 \mathrm{mg} / \mathrm{dl}$ ) or severe nocturnal hypoglycemia was similar between the two groups (Table 2). The rate of confirmed (plasma glucose $<70 \mathrm{mg} / \mathrm{dl}$ ) or severe nocturnal 
hypoglycemia was lower in the Gla-300 group than in the Gla-100 group during the first 8 weeks of the study (rate ratio $0.69,95 \%$ CI 0.53 0.91) [63]. Over the whole study period, the incidence of confirmed or severe hypoglycemia at any time was similar between treatment groups (Table 2). Severe hypoglycemia was seen in $6.6 \%$ and $9.5 \%$ of patients in Gla-300 and Gla-100 groups, respectively. Neither glycemic control nor hypoglycemia differed between insulins or times for morning and evening injection.

Similarly to the EDITION JP 2 trial, EDITION JP 1 was a 26-week, randomized, open-label study conducted with Japanese participants who received either Gla-300 or Gla-100 alongside continued use of mealtime insulin [64]. Gla-300 showed similar efficacy to Gla-100 in terms of lowering A1C. The incidence of confirmed or severe nocturnal hypoglycemia was not significantly different between groups from Week 9 to Week 26; however, the incidence was lowest during the first eight weeks of the study in the Gla-300 group compared with the Gla100 group, and was lower in the Gla-300 group over the full 26-week study period (Table 2). Severe hypoglycemia was low in both groups (5.7\% and 9.9\% with Gla-300 and Gla-100, respectively). There was no difference in the incidence of confirmed or severe hypoglycemia experienced at any time between groups (Table 2).

LY2605541, basal insulin peglispro: There are data from one 8-week, phase 2, open-label, randomized, two-arm, cross-over study in patients with T1DM who received either BIL or Gla-100 QD while continuing mealtime insulin [65]. In this study, BIL demonstrated greater improvements compared with Gla-100 in terms of glycemic control. The rate of total hypoglycemia was higher in the BIL group than in the Gla-100 group (Table 2). However, the rate of nocturnal hypoglycemia was lower in the BIL group than in the Gla-100 group (Table 2). The incidence of severe hypoglycemia was similar between the two treatment groups (five patients with six events in the BIL group and three patients with six events in the Gla-100 group).

Two phase 3 trials comparing BIL to Gla-100 or NPH have been completed as part of the IMAGINE trial series, with reporting of results expected shortly (NCT01481779, NCT01454284; https://clinicaltrials. gov).

New insulin LY2963016: The ELEMENT 1 trial was a 52-week, phase 3, open-label, parallel-group study in which patients received either LY2963016 or Gla-100 in combination with mealtime insulin lispro [66]. Insulin doses were titrated to achieve blood glucose $\geq$ or $\sim 110 \mathrm{mg} / \mathrm{dl}(\leq 6.0 \mathrm{mmol} / \mathrm{l})$. LY2963016 had similar efficacy to Gla100 in terms of lowering $\mathrm{A} 1 \mathrm{C}$, and the rate of total hypoglycemia was similar between patient groups (Table 2).

\section{Summary}

The longer, more constant PK/PD profiles of the new basal analog insulins appear to confer advantages over previous basal analog insulins with respect to reduced hypoglycemia, particularly nocturnal hypoglycemia. However, interpretation of the data is limited by a lack of head-to-head comparisons between these agents and the fact that all data published to date are from trials sponsored by the pharmaceutical company producing the insulin, with no independent meta-analyses currently available. In addition, the trials have been designed to evaluate efficacy outcomes and are thus powered to detect differences in $\mathrm{A} 1 \mathrm{C}$ rather than hypoglycemia. Hence, they might be underpowered to detect differences in hypoglycemia. Indeed, a meta-analysis of three T2DM trials showed a reduction in overall confirmed or severe hypoglycemia when using Gla-300 compared with Gla-100, which was not consistently observed in the individual clinical trials included in the meta-analysis [52].

There remains a lack of consistent definitions and outcome measures for hypoglycemia and patient selection criteria in trials of insulin therapies, which adds an unwelcome layer of complexity for clinicians wishing to make descriptive comparisons of hypoglycemia rates between trials. Lack of standardized reporting underlines the need for head-to-head trials and subsequent meta-analyses of data. Trials designed specifically to assess the effect of novel basal analog insulins in patients with a history of hypoglycemia unawareness or at high risk for severe hypoglycemia should also be undertaken. The difficulty in performing double-blind assessments in insulin trials, due to the different appearances of formulations, is a perennial issue in randomized trials comparing basal analog insulins. However, two of the upcoming phase 3 studies of BIL have a double-blind design (NCT01435616, NCT01454284; http://clinicaltrials.gov/). Whether this represents a crossing of the Rubicon for the design of insulin trials remains to be seen.

\section{Conclusion}

The development of the new generation of basal analog insulins represents an additional step towards patients achieving physiologic glycemic control. Improved PK/PD profiles appear to be associated with better clinical outcomes in terms of hypoglycemia. Future head-to-head trials, studies in specific patient populations, and pharmacoeconomic analyses - many of which are already underway - will be key for clinicians and patients to determine appropriate, individualized treatment courses.

\section{Acknowledgments}

The contents of this paper and the opinions expressed within are those of the authors, and it was the decision of the authors to submit the manuscript for publication. The authors contributed to the writing of this manuscript, including critical review and editing of each draft, and approval of the submitted version. The authors received writing/editorial support in the preparation of this manuscript provided by Pim Dekker, PhD, of Excerpta Medica, funded by Sanofi US, Inc.

\section{Conflicts of Interest}

\section{Alsahli: None}

Thrasher: Research grant support from Amgen, Aventis, Boehringer Ingelheim, Bristol-Myers Squibb, Eli Lilly, Janssen, Gilead Sciences, GlaxoSmithKline Medtronic, Merck \& Co, Novartis, Novo Nordisk, Pfizer, Sanofi, Speedel Pharma Ltd, and Yamanouchi Pharma America; has served on the speaker bureau for Amylin, AstraZeneca, Boehringer Ingelheim, Bristol-Myers Squibb, Daiichi-Sankyo GlaxoSmithKline, Janssen, Lilly, Medtronic, Novo Nordisk, Pfizer, Sanofi, Takeda, and Virus, and is on advisory boards for Medtronic, Pfizer Pharmaceuticals, and Sanofi-Aventis; and has received editorial/publication support from Boehringer Ingelheim and Eli Lilly.

Gerich: Consultant/member of the speaker bureau for Bristol-Myers Squibb, AstraZeneca, Merck, Janssen Pharmaceuticals, Eli Lilly, and Boehringer Ingelheim.

\section{References}

1. Inzucchi SE, Bergenstal RM, Buse JB, Diamant M, Ferrannini E, et al. (2015) Management of hyperglycemia in type 2 diabetes, 2015: a patient-centered approach: update to a position statement of the American Diabetes Association and the European Association for the Study of Diabetes. Diabetes Care. 38 : 140-149.

2. Mitrakou A, Ryan C, Veneman T, Mokan M, Jenssen T, et al. (1991) Hierarchy of glycemic thresholds for counterregulatory hormone secretion, symptoms, and cerebral dysfunction. Am J Physiol 260: E67-74.

3. Gerich JE (1988) Lilly lecture 1988. Glucose counterregulation and its impact on diabetes mellitus. Diabetes 37: 1608-1617.

4. Alsahli M, Gerich JE (2013) Hypoglycemia. Endocrinol Metab Clin North Am 42: 657-676. 
Citation: Alsahli M, Thrasher JR, Gerich JE (2015) Hypoglycemia with New-Generation Basal Analog Insulins: A Descriptive Critical Review. J Diabetes Metab 6: 576. doi:10.4172/2155-6156.1000576

5. Bolli GB, Tsalikian E, Haymond MW, Cryer PE, Gerich JE (1984) Defective glucose counterregulation after subcutaneous insulin in noninsulin-dependent diabetes mellitus. Paradoxical suppression of glucose utilization and lack of compensatory increase in glucose production, roles of insulin resistance, abnormal neuroendocrine responses, and islet paracrine interactions. J Clin Invest 73: 1532-1541.

6. Gerich JE, Mokan M, Veneman T, Korytkowski M, Mitrakou A (1991) Hypoglycemia unawareness. Endocr Rev 12: 356-371.

7. Fritsche A, Stumvoll M, Häring HU, Gerich JE (2000) Reversal of hypoglycemia unawareness in a long-term type 1 diabetic patient by improvement of betaadrenergic sensitivity after prevention of hypoglycemia. J Clin Endocrinol Metab 85: 523-525.

8. Holman RR, Paul SK, Bethel MA, Matthews DR, Neil HA (2008) 10-year followup of intensive glucose control in type 2 diabetes. N Engl J Med 359: 1577 1589.

9. Diabetes Control and Complications Trial Research Group (1993) The effect of intensive treatment of diabetes on the development and progression of long-term complications in insulin-dependent diabetes mellitus. The Diabetes Control and Complications Trial Research Group. N Engl J Med 329: 977-986.

10. UK Prospective Diabetes Study (UKPDS) Group (1998) Intensive bloodglucose control with sulphonylureas or insulin compared with conventional treatment and risk of complications in patients with type 2 diabetes (UKPDS 33). Lancet 352: 837-853. Erratum in: Lancet 1999; 354: 837-853.

11. Action to Control Cardiovascular Risk in Diabetes Study Group, Gerstein HC Miller ME, Byington RP, Goff DC Jr, et al. (2008) Effects of intensive glucose lowering in type 2 diabetes. N Engl J Med 358: 2545-2559.

12. Currie CJ, Morgan CL, Poole CD, Sharplin P, Lammert M, et al. (2006) Multivariate models of health-related utility and the fear of hypoglycaemia in people with diabetes. Curr Med Res Opin 22: 1523-1534

13. Leiter LA, Yale J-F, Chiasson J-L, Harris S, Kleinstiver $P$, et al. (2005) Assessment of the impact of fear of hypoglycemic episodes on glycemic and hypoglycemia management. Can J Diabetes 29:186-192.

14. Brod M, Rana A, Barnett AH (2012) Adherence patterns in patients with type 2 diabetes on basal insulin analogues: missed, mistimed and reduced doses. Curr Med Res Opin 28: 1933-1946.

15. Wild D, von Maltzahn R, Brohan E, Christensen T, Clauson P, et al. (2007) A critical review of the literature on fear of hypoglycemia in diabetes: Implications for diabetes management and patient education. Patient Educ Couns 68: 1015.

16. Grant R, Adams AS, Trinacty CM, Zhang F, Kleinman K, et al. (2007) Relationship between patient medication adherence and subsequent clinical inertia in type 2 diabetes glycemic management. Diabetes Care 30:807-812.

17. Nam S, Chesla C, Stotts NA, Kroon L, Janson SL (2011) Barriers to diabetes management: patient and provider factors. Diabetes Res Clin Pract 93: 1-9.

18. Woerle HJ, Neumann C, Zschau S, Tenner S, Irsigler A, et al. (2007) Impact of fasting and postprandial glycemia on overall glycemic control in type 2 diabetes. Importance of postprandial glycemia to achieve target $\mathrm{HbA} 1 \mathrm{c}$ levels. Diabetes Res Clin Pract 77: 280-285.

19. Ratner RE, Hirsch IB, Neifing JL, Garg SK, Mecca TE, et al. (2000) Less hypoglycemia with insulin glargine in intensive insulin therapy for type 1 diabetes. U.S. Study Group of Insulin Glargine in Type 1 Diabetes. Diabetes Care 23: 639-643.

20. Riddle MC, Rosenstock J, Gerich J; Insulin Glargine 4002 Study Investigators (2003) The treat-to-target trial: randomized addition of glargine or human NPH insulin to oral therapy of type 2 diabetic patients. Diabetes Care 26: 3080-3086.

21. Strange $P$ (2007) Treat-to-target insulin titration algorithms when initiating long or intermediate acting insulin in type 2 diabetes. J Diabetes Sci Technol 1 : 540-548.

22. Garber AJ1 (2014) Treat-to-target trials: uses, interpretation and review of concepts. Diabetes Obes Metab 16: 193-205.

23. Wangnoo SK, Sethi B, Sahay RK, John M, Ghosal S, et al. (2014) Treat-totarget trials in diabetes. Indian J Endocrinol Metab 18: 166-174.

24. Rizza RA, Gerich JE, Haymond MW, Westland RE, Hall LD, et al. (1980) Control of blood sugar in insulin-dependent diabetes: comparison of an artificial endocrine pancreas, continuous subcutaneous insulin infusion, and intensified conventional insulin therapy. N Engl J Med 303: 1313-1318.
25. Heise T, Pieber TR (2007) Towards peakless, reproducible and long-acting insulins. An assessment of the basal analogues based on isoglycaemic clamp studies. Diabetes Obes Metab 9: 648-659.

26. Swinnen SG, Simon AC, Holleman F, Hoekstra JB, Devries JH (2011) Insulin detemir versus insulin glargine for type 2 diabetes mellitus. Cochrane Database Syst Rev 7: CD006383.

27. Heise T, Nosek L, Bøttcher SG, Hastrup H, Haahr H (2012) Ultra-long-acting insulin degludec has a flat and stable glucose-lowering effect in type 2 diabetes. Diabetes Obes Metab 14: 944-950.

28. Heller S, Buse J, Fisher M, Garg S, Marre M, et al. (2012) Insulin degludec, an ultra-longacting basal insulin, versus insulin glargine in basal-bolus treatment with mealtime insulin aspart in type 1 diabetes (BEGIN Basal-Bolus Type 1): a phase 3 , randomised, open-label, treat-to-target non-inferiority trial. Lancet 379: 1489-1497.

29. Becker RH, Dahmen R, Bergmann K, Lehmann A, Jax T, et al. (2015) New insulin glargine 300 Units $\cdot \mathrm{mL}-1$ provides a more even activity profile and prolonged glycemic control at steady state compared with insulin glargine 100 Units $\cdot \mathrm{mL}^{-1}$. Diabetes Care 38: 637-643

30. Shiramoto M, Eto T, Irie S, Fukuzaki A, Teichert L, et al. (2015) Single-dose new insulin glargine $300 \mathrm{U} / \mathrm{ml}$ provides prolonged, stable glycaemic control in Japanese and European people with type 1 diabetes. Diabetes Obes Metab 17: $254-260$

31. Dahmen R, Bergmann K, Lehmann A, Tillner J, Jax T, et al. (2013) New insulin glargine U300 formulation evens and prolongs steady state PK and PD profiles during euglycemic clamp in patients with type 1 diabetes (T1DM). Diabetes 62: A113.

32. Steinstraesser A, Schmidt R, Bergmann K, Dahmen R, Becker RH (2014) Investigational new insulin glargine $300 \mathrm{U} / \mathrm{ml}$ has the same metabolism as insulin glargine $100 \mathrm{U} / \mathrm{ml}$. Diabetes Obes Metab 16: 873-876.

33. Beals JM, Cutler GB, Vick A, Koester A, Li S, et al. (2012) LY2605541 Leveraging hydrodynamic size to develop a novel basal insulin. Diabetologia 55: S23.

34. Sinha VP, Howey DC, Choi SL, Mace KF, Heise T (2014) Steady-state pharmacokinetics and glucodynamics of the novel, long-acting basal insulin LY2605541 dosed once-daily in patients with type 2 diabetes mellitus. Diabetes Obes Metab 16: 344-350.

35. Linnebjerg H, Heise T, Zhang X, Seger ME, Coutant D, et al. (2014) Comparison of duration of action of 2 insulin glargine products, LY2963016 and insulin glargine, in subjects with type 1 diabetes mellitus. Diabetologia 57: S382.

36. Choi I, Hwang S, Kim J, Jung S, Kim D, et al. (2014) Long-acting basal insulin (HM12470) offers once-weekly dosing potential with a favorable PK, PD and mitogenic profile. Diabetologia 57:1 S381.

37. Kaye J, Krasner A, Canney L, Pichotta P, Simms P, et al. (2013) Nove formulations BIOD-238 and BIOD-250 result in more rapid absorption and declines from peak than Humalog. Diabetologia 56: S413.

38. Morrow L, Hompesch M, Canney L, Pichotta P, Krasner A, et al. (2014) Biphasic pharmacokinetic and pharmacodynamic profiles associated with concentrated insulin BIOD-531 show rapid onset and basal duration of action. Diabetologia 57: S383.

39. Frier BM (2014) Hypoglycaemia in diabetes mellitus: epidemiology and clinical implications. Nat Rev Endocrinol 10: 711-722.

40. Gerich JE (2000) Hypoglycaemia and counterregulation in type 2 diabetes. Lancet 356: 1946-1947.

41. Wei W, Zhou S, Miao R, Pan C, Xie L, et al. (2014) Much ado about nothing? A real-world study of patients with type 2 diabetes switching Basal insulin analogs. Adv Ther 31: 539-560.

42. Morales J, Schneider D2 (2014) Hypoglycemia. Am J Med 127: S17-24.

43. FDA briefing document (2013) NDA 203313 and NDA 203314. Insulin Degludec and Insulin Degludec/Aspart.

44. Novo Nordisk (2013) Novo Nordisk receives Complete Response Letter in the US for Tresiba ${ }^{\circledR}$ and Ryzodeg $®$.

45. Vora J, Christensen T, Rana A, Bain SC (2014) Insulin degludec versus insulin glargine in type 1 and type 2 diabetes mellitus: a meta-analysis of endpoints in phase 3a trials. Diabetes Ther 5: 435-446. 
Citation: Alsahli M, Thrasher JR, Gerich JE (2015) Hypoglycemia with New-Generation Basal Analog Insulins: A Descriptive Critical Review. J Diabetes Metab 6: 576. doi:10.4172/2155-6156.1000576

Page 9 of 9

46. Zinman B, Philis-Tsimikas A, Cariou B, Handelsman Y, Rodbard HW, et al. (2012) Insulin degludec versus insulin glargine in insulin-naive patients with type 2 diabetes: a 1-year, randomized, treat-to-target trial (BEGIN Once Long). Diabetes Care 35: 2464-2471.

47. Garber AJ, King AB, Del Prato S, Sreenan S, Balci MK, et al. (2012) Insulin degludec, an ultra-longacting basal insulin, versus insulin glargine in basalbolus treatment with mealtime insulin aspart in type 2 diabetes (BEGIN BasalBolus Type 2): a phase 3, randomised, open-label, treat-to-target non-inferiority trial. Lancet 379: 1498-1507.

48. Meneghini L, Atkin SL, Gough SC, Raz I, Blonde L, et al. (2013) The efficacy and safety of insulin degludec given in variable once-daily dosing intervals compared with insulin glargine and insulin degludec dosed at the same time daily: a 26-week, randomized, open-label, parallel-group, treat-to-target trial in individuals with type 2 diabetes. Diabetes Care 36: 858-864.

49. Onishi Y, Iwamoto Y, Yoo SJ, Clauson P, Tamer SC, et al. (2013) Insulin degludec compared with insulin glargine in insulin-naïve patients with type 2 diabetes: a 26-week, randomized, controlled, Pan-Asian, treat-to-target trial. J Diabetes Investig 4: 605-612.

50. Sanofi (2015) FDA accepts Sanofi's new drug application for basal insulin Toujeo®. Paris, France.

51. EMA (2015) Summary of opinion (post authorization). Toujeo: insulin glargine 300 units $/ \mathrm{ml}$.

52. Ritzel RA, Roussel R, Bolli GB, Vinet L, Yki-Järvinen H (2014) New insulin glargine $300 \mathrm{U} / \mathrm{ml}$ : glycaemic control and hypoglycaemia in a meta-analysis of phase 3a EDITION clinical trials in people with type 2 diabetes mellitus. Diabetologia 57: S394-S395.

53. American Diabetes Association (2015) Standards of medical care in diabetes-2015. Diabetes Care 38: S1-S92.

54. Riddle MC, Bolli GB, Ziemen M, Muehlen-Bartmer I, Bizet F, et al. (2014) New insulin glargine 300 units $/ \mathrm{mL}$ versus glargine $100 \mathrm{units} / \mathrm{mL}$ in people with type 2 diabetes using basal and mealtime insulin: glucose control and hypoglycemia in a 6-month randomized controlled trial (EDITION 1). Diabetes Care. 37: 27552762.

55. Yki-Järvinen $\mathrm{H}$, Bergenstal $\mathrm{R}$, Ziemen $\mathrm{M}$, Wardecki M, Muehlen-Bartmer I, et al. (2014) New insulin glargine $300 \mathrm{U} / \mathrm{mL}$ versus glargine $100 \mathrm{U} / \mathrm{mL}$ in people with type 2 diabetes using oral agents and basal insulin: glucose control and hypoglycemia in a 6-month randomized controlled trial (EDITION 2). Diabetes Care 37: 3235-3243.

56. Bolli GB, Riddle MC, Bergenstal RM, Ziemen M, Sestakauskas K, et al. (2015)
New insulin glargine $300 \mathrm{U} / \mathrm{ml}$ compared with glargine $100 \mathrm{U} / \mathrm{ml}$ in insulin-naïve people with type 2 diabetes on oral glucose-lowering drugs: a randomized controlled trial (EDITION 3). Diabetes Obes Metab 17: 386-394.

57. Terauchi Y, Koyama M, Cheng X, Shimizu S, Hirose T, et al. (2014) Glycaemic control and hypoglycaemia in Japanese people with T2DM receiving new insulin glargine $300 \mathrm{U} / \mathrm{mL}$ in combination with OADs (EDITION JP 2). Diabetologia 57: S401.

58. Bergenstal RM, Rosenstock J, Arakaki RF, Prince MJ, Qu Y, et al. (2012) A randomized, controlled study of once-daily LY2605541, a novel long-acting basal insulin, versus insulin glargine in basal insulin-treated patients with type 2 diabetes. Diabetes Care 35:2140-2147.

59. European Medicines Agency (2014) Summary of opinion (initial authorisation) Abasria (insulin glargine)

60. Eli Lilly and Company (2014) FDA grants tentative approval for Lilly and Boehringer Ingelheim's Basaglar ${ }^{\mathrm{TM}}$ (insulin glargine injection).

61. Hollander P, Rosenstock J, Bhargava A, llag LL, Pollom RK, et al. (2014) Similar efficacy and safety with LY2963016 insulin glargine compared with insulin glargine in patients with type 2 diabetes mellitus: the ELEMENT 2 study. Diabetologica 57: S388.

62. Mathieu C, Hollander P, Miranda-Palma B, Cooper J, Franek E, et al. (2013) Efficacy and safety of insulin degludec in a flexible dosing regimen vs insulin glargine in patients with type 1 diabetes (BEGIN: Flex T1): a 26-week randomized, treat-to-target trial with a 26 -week extension. J Clin Endocrinol Metab 98: 1154-1162.

63. Home PD, Bergenstal RM, Riddle MC, Ziemen M, Rojeski M, et al. (2014) Glycaemic control and hypoglycaemia with new insulin glargine $300 \mathrm{U} / \mathrm{mL}$ in people with type 1 diabetes (EDITION 4). Diabetologia 57: S69-S70.

64. Matsuhisa M, Koyama M, Cheng X, Shimizu S, Hirose T (2014) New insulin glargine $300 \mathrm{U} / \mathrm{mL}$ : glycaemic control and hypoglycaemia in Japanese people with T1DM (EDITION JP 1). Diabetologia 57: S400.

65. Rosenstock J, Bergenstal RM, Blevins TC, Morrow LA, Prince MJ, et al. (2013) Better glycemic control and weight loss with the novel long-acting basal insulin LY2605541 compared with insulin glargine in type 1 diabetes: a randomized crossover study. Diabetes Care 36: 522-528.

66. Blevins TC, Dahl D, Rosenstock J, llag LL, Huster WJ, et al. (2015) Efficacy and safety of LY2963016 insulin glargine compared with insulin glargine (Lantus $®$ ) in patients with type 1 diabetes in a randomized controlled trial: the ELEMENT 1 study. Diabetes Obes Metab.
Citation: Hypoglycemia with New-Generation Basal Analog Insulins: A Descriptive Critical Review. J Diabetes Metab 6: 576. doi:10.4172/2155 6156.1000576
Submit your next manuscript and get advantages of OMICS Group submissions

Unique features:

- Increased global visibility of articles through worldwide distribution and indexing

- Showcasing recent research output in a timely and updated manner

Special issues on the current trends of scientific research

Special features:

- 700 Open Access Journals

50,000 Editorial team

Rapid review process

- Quality and quick editorial, review and publication processing

- Indexing at PubMed (partial), Scopus, EBSCO, Index Copernicus, Google Scholar etc.

- Sharing Option: Social Networking Enabled

- Authors, Reviewers and Editors rewarded with online Scientific Credits

- Better discount for your subsequent articles

Submit your manuscript at: http://www.omicsonline.org/submission 